\title{
Advanced functionality for radio analysis in the Offline software framework of the Pierre Auger Observatory
}

P. Abreu ${ }^{\text {bk }}$, M. Aglietta ${ }^{a x}$, E.J. Ahn ${ }^{\text {bz }}$, I.F.M. Albuquerque ${ }^{\mathrm{n}, \mathrm{bz}}$, D. Allard ${ }^{\mathrm{aa}}$, I. Allekotte ${ }^{\mathrm{a}}$, J. Allen ${ }^{\mathrm{cc}}$, P. Allison ${ }^{c e}$, J. Alvarez Castillo ${ }^{\text {bd }}$, J. Alvarez-Muñiz ${ }^{\text {br }}$, M. Ambrosio ${ }^{a r}$, A. Aminaei ${ }^{\text {be }}$, L. Anchordoqui ${ }^{\mathrm{cl}}$, S. Andringa bk, T. Antičić ${ }^{\mathrm{v}}$, C. Aramo ${ }^{\mathrm{ar}}$, E. Arganda ${ }^{\text {bo }}$, F. Arqueros ${ }^{\text {bo }}$, H. Asorey ${ }^{\mathrm{a}}$, P. Assis ${ }^{\text {bk }}$, J. Aublin ${ }^{\text {ac }}$, M. Ave ai,ag, M. Avenier ${ }^{\text {ad }}$, G. Avila ${ }^{\mathrm{i}}$, T. Bäcker ${ }^{\mathrm{am}}$, M. Balzer $^{\mathrm{ah}}$, K.B. Barber ${ }^{\mathrm{j}}$, A.F. Barbosa $^{\mathrm{k}}$, R. Bardenet ${ }^{\mathrm{ab}}$, S.L.C. Barroso ${ }^{\mathrm{q}}$, B. Baughman ${ }^{\text {ce }}$, J.J. Beatty ${ }^{\mathrm{ce}}$, B.R. Becker ${ }^{\mathrm{cj}}$, K.H. Becker ${ }^{\text {af }}$, J.A. Bellido ${ }^{\mathrm{j}}$, S. BenZvi ${ }^{\mathrm{ck}}$, C. Berat $^{\text {ad }}$, X. Bertou ${ }^{\text {a }}$, P.L. Biermann ${ }^{\text {aj }}$, P. Billoir ${ }^{\text {ac }}$, F. Blanco ${ }^{\text {bo }}$, M. Blanco $^{\text {bp }}$, C. Bleve ${ }^{\text {af }}$, H. Blümer $^{\text {ai,ag }}$, M. Boháčová ${ }^{\mathrm{x}, \mathrm{ch}}$, D. Boncioli as, C. Bonifazi ${ }^{\text {t,ac }}$, R. Bonino ${ }^{\mathrm{ax}}, \mathrm{N}$. Borodai ${ }^{\mathrm{bi}}$, J. Brack ${ }^{\mathrm{bx}}$, P. Brogueira $^{\text {bk }}$, W.C. Brown ${ }^{\text {by }}$, R. Bruijn ${ }^{\text {bt }}$, P. Buchholz ${ }^{\text {am }}$, A. Bueno ${ }^{\text {bq }}$, R.E. Burton ${ }^{\text {bv }}$, K.S. Caballero-Mora ${ }^{\text {ai }}$, L. Caramete ${ }^{\text {aj }}$, R. Caruso ${ }^{\text {at }}$, A. Castellina ${ }^{\text {ax }}$, G. Cataldi aq, L. Cazon ${ }^{\text {bk }}$, R. Cester ${ }^{\text {au }}$, J. Chauvin ${ }^{\text {ad }}$, A. Chiavassa ${ }^{a x}$, J.A. Chinellato ${ }^{\circ}, A$. Chou ${ }^{\text {bz,cc }}{ }^{\text {, J. Chudoba }}{ }^{\mathrm{x}}$, R.W. Clay ${ }^{\mathrm{j}}$, M.R. Coluccia ${ }^{\text {aq }}$, R. Conceição ${ }^{\text {bk }}$, F. Contreras ${ }^{\text {h }}$, H. Cook ${ }^{\text {bt }}$, M.J. Cooper ${ }^{\mathrm{j}}$, J. Coppens ${ }^{\text {be,bg }}$, A. Cordier ${ }^{\mathrm{ab}}$, U. Cotti ${ }^{\text {bc }}$, S. Coutu ${ }^{\text {cf }}$, C.E. Covault $^{\text {bv }}$, A. Creusot ${ }^{\text {aa,bm }}$, A. Criss ${ }^{\text {cf }}$, J. Cronin ${ }^{\text {ch }}$, A. Curutiu ${ }^{\text {aj }}$, S. Dagoret-Campagne ${ }^{\text {ab }}$, R. Dallier ${ }^{\text {ae }}$, S. Dasso ${ }^{\text {f,d }}$, K. Daumiller ${ }^{\text {ag }}$, B.R. Dawson ${ }^{\mathrm{j}}$, R.M. de Almeida ${ }^{\mathrm{u}, \mathrm{o}}$, M. De Domenico ${ }^{\text {at }}$, C. De Donato ${ }^{\text {bd,ap }}$, S.J. de Jong ${ }^{\text {be }}$, G. De La Vega ${ }^{g}$, W.J.M. de Mello Junior ${ }^{\circ}$, J.R.T. de Mello Neto ${ }^{t}$, I. De Mitri ${ }^{a q}$, V. de Souza ${ }^{\mathrm{m}}$, K.D. de Vries ${ }^{\text {bf }}$, G. Decerprit ${ }^{a}$, L. del Peral ${ }^{\text {bp }}$, O. Deligny ${ }^{z}$, H. Dembinski $^{a i, a g}$, A. Denkiewicz ${ }^{b}$, C. Di Giulio ${ }^{\text {ao,as }}$, J.C. Diaz ${ }^{\text {cb }}$, M.L. Díaz Castro ${ }^{1}$, P.N. Diep ${ }^{\text {l,cm }}$, C. Dobrigkeit ${ }^{\circ}$, J.C. D’Olivo ${ }^{\text {bd }}$, P.N. Dong ${ }^{\text {cm,z }}$, A. Dorofeev ${ }^{\text {bx }}$, J.C. dos Anjos ${ }^{k}$, M.T. Dova ${ }^{\mathrm{e}}$, D. D’Urso ${ }^{\mathrm{ar}}$, I. Dutan ${ }^{\mathrm{aj}}, \mathrm{J}_{\text {. Ebr }}^{\mathrm{x}}$, R. Engel ${ }^{\mathrm{ag}}$, M. Erdmann ${ }^{\text {ak }}$, C.O. Escobar ${ }^{\circ}$, A. Etchegoyen ${ }^{\text {b }}$, P. Facal San Luis ${ }^{\text {ch }}$, H. Falcke ${ }^{\text {be,bh }}$, G. Farrar ${ }^{\text {cc }}$, A.C. Fauth ${ }^{\text {o }}$, N. Fazzini $^{\text {bz }}$, A.P. Ferguson $^{\text {bv }}$, A. Ferrero ${ }^{\text {b }}$, B. Fick ${ }^{\text {cb }}$, A. Filevich ${ }^{\text {b }}$, A. Filipčič ${ }^{\text {bl,bm }}{ }^{\text {, S. Fliescher }}{ }^{\text {ak }}$, C.E. Fracchiolla ${ }^{\text {bx }}$, E.D. Fraenkel ${ }^{\text {bf }}$, U. Fröhlich ${ }^{\text {am }}$, B. Fuchs ${ }^{\text {k }}$, R.F. Gamarra ${ }^{\text {b }}$, S. Gambetta ${ }^{\text {an }}$, B. García ${ }^{\text {g }}$, D. García Gámez ${ }^{\text {bq }}$, D. Garcia-Pinto $^{\text {bo }}$, A. Gascon ${ }^{\text {bq }}$, H. Gemmeke ${ }^{\text {ah }}$, K. Gesterling ${ }^{\text {cj }}$, P.L. Ghia ${ }^{\text {ac,ax }}$, U. Giaccari ${ }^{\text {aq }}$, M. Giller ${ }^{\text {bj' }}$, H. Glass ${ }^{\text {bz }}$, M.S. Gold ${ }^{c j}$, G. Golup ${ }^{a}$, F. Gomez Albarracin ${ }^{\mathrm{e}}$, M. Gómez Berisso ${ }^{\mathrm{a}}$, P. Gonçalves ${ }^{\mathrm{bk}}$, D. Gonzalez ai, J.G. Gonzalez ai, B. Gookin ${ }^{\text {bx }}$, D. Góra ${ }^{\text {ai,bi }}$, A. Gorgi ax, P. Gouffon ${ }^{\text {n }}$, S.R. Gozzini bt, E. Grashorn ${ }^{\text {ce }}$, S. Grebe ${ }^{\text {be }}$, N. Griffith ${ }^{\text {ce }}$, M. Grigat ${ }^{\text {ak }}$, A.F. Grillo ${ }^{a y}$, Y. Guardincerri ${ }^{\text {d }}$, F. Guarino ${ }^{\text {ar }}$, G.P. Guedes $^{\text {p }}$, J.D. Hague ${ }^{\text {cj }}$, P. Hansen ${ }^{\text {e }}$, D. Harari ${ }^{\text {a }}$, S. Harmsma ${ }^{\text {bf,bg }}{ }^{\text {, J.L. Harton }}{ }^{\text {bx }}$, A. Haungs ${ }^{\text {ag }}$, T. Hebbeker ${ }^{\text {ak }}$, D. Heck ${ }^{\text {ag }}$, A.E. Herve ${ }^{j}$, C. Hojvat $^{\text {bz }}$, V.C. Holmes ${ }^{j}$, P. Homola ${ }^{\text {bi }}$, J.R. Hörandel ${ }^{\text {be }}$, A. Horneffer ${ }^{\text {be }}$, M. Hrabovský ${ }^{\mathrm{x}, \mathrm{y}}, \mathrm{T}$. Huege ${ }^{\mathrm{ag}}, \mathrm{A}$. Insolia ${ }^{\text {at }}, \mathrm{F}$. Ionita ${ }^{\mathrm{ch}}, \mathrm{A}$. Italiano ${ }^{\text {at }}, \mathrm{S}$. Jiraskova ${ }^{\text {be }}$, K. Kadija ${ }^{\text {v }}$, K.H. Kampert ${ }^{\text {af,* }}$, P. Karhan ${ }^{\text {w }}$, T. Karova ${ }^{\text {x }}$, P. Kasper ${ }^{\text {bz }}$, B. Kégl ${ }^{\text {ab }}$, B. Keilhauer ${ }^{\text {ag }}$, A. Keivani $^{\text {ca }}$, J.L. Kelley ${ }^{\text {be }}$, E. Kemp ${ }^{\text {o }}$, R.M. Kieckhafer ${ }^{\text {cb }}$, H.O. Klages ${ }^{\text {ag }}$, M. Kleifges ${ }^{\text {ah }}$, J. Kleinfeller ${ }^{\text {ag }}$, J. Knapp ${ }^{\text {bt }}$, D.-H. Koang ${ }^{\text {ad }}$, K. Kotera ${ }^{\text {ch }}$, N. Krohm ${ }^{\text {af }}$, O. Krömer ${ }^{\text {ah }}$, D. Kruppke-Hansen af, F. Kuehn bz, D. Kuempel ${ }^{\text {af }}$, J.K. Kulbartz ${ }^{\text {al }}$, N. Kunka ${ }^{\text {ah }}$, G. La Rosa ${ }^{\text {aw }}$, C. Lachaud ${ }^{\text {aa }}$, P. Lautridou ${ }^{\text {ae }}$, M.S.A.B. Leão ${ }^{\text {, }}$, D. Lebrun ${ }^{\text {ad }}$, P. Lebrun ${ }^{b z}$, M.A. Leigui de Oliveira ${ }^{s}$, A. Lemiere ${ }^{z}$, A. Letessier-Selvon ${ }^{\text {ac }}$, I. Lhenry-Yvon ${ }^{z}, K$. Link ${ }^{\text {ai }}$, R. López ${ }^{\text {ba }}$, A. Lopez Agüera ${ }^{\text {br }}$, K. Louedec $^{\text {ab }}$, J. Lozano Bahilo ${ }^{\text {bq }}$, A. Lucero ${ }^{\text {b,ax }}$, M. Ludwig ${ }^{\text {ai }}$, H. Lyberis ${ }^{\text {, }}$, C. Macolino $^{\text {ac }}$, S. Maldera ${ }^{a x}$, D. Mandat ${ }^{\mathrm{x}}$, P. Mantsch ${ }^{\text {bz }}$, A.G. Mariazzi ${ }^{\text {e }}$, V. Marin ${ }^{\text {ae }}$, I.C. Maris ${ }^{\text {ac }}$, H.R. Marquez Falcon ${ }^{\text {bc }}$, G. Marsella ${ }^{\text {av }}$, D. Martello ${ }^{\mathrm{aq}}$, L. Martin ${ }^{\mathrm{ae}}$, O. Martínez Bravo ${ }^{\text {ba }}$, H.J. Mathes ${ }^{\text {ag }}$, J. Matthews $^{\text {ca,cg }}$, J.A.J. Matthews ${ }^{\text {cj }}$, G. Matthiae ${ }^{\text {as }}$, D. Maurizio ${ }^{\text {au }}$, P.O. Mazur ${ }^{\text {bz }}$, G. Medina-Tanco ${ }^{\text {bd }}$, M. Melissas ${ }^{a i}$, D. Melo ${ }^{\text {b,au }}$, E. Menichetti ${ }^{\text {au }}$, A. Menshikov ${ }^{\text {ah }}$, P. Mertsch ${ }^{\text {bs }}$, C. Meurer ${ }^{\text {ak }}$, S. Mićanović ${ }^{v}$, M.I. Micheletti ${ }^{\mathrm{b}}$, W. Miller ${ }^{\mathrm{cj}}$, L. Miramonti ${ }^{\mathrm{ap}}$, S. Mollerach $^{\mathrm{a}}$, M. Monasor ${ }^{\mathrm{ch}}$, D. Monnier Ragaigne ${ }^{\mathrm{ab}}$, F. Montanet ${ }^{\mathrm{ad}}$, B. Morales ${ }^{\mathrm{bd}}$, C. Morello $^{\mathrm{ax}}$, E. Moreno ${ }^{\text {ba }}$, J.C. Moreno $^{\mathrm{e}}$, C. Morris ${ }^{\text {ce }}$, M. Mostafá $^{\mathrm{bx}}$,

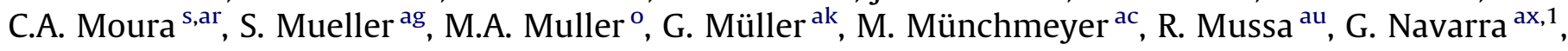


J.L. Navarro ${ }^{\text {bq }}$, S. Navas ${ }^{\text {bq }}$, P. Necesal ${ }^{\mathrm{x}}$, L. Nellen ${ }^{\text {bd }}$, A. Nelles ${ }^{\text {be,ak }}{ }^{\text {, P.T. Nhung }}{ }^{\text {l,cm }}$, N. Nierstenhoefer ${ }^{\text {af }}$, D. Nitz ${ }^{\mathrm{cb}}$, D. Nosek ${ }^{\mathrm{w}}$, L. Nožka ${ }^{\mathrm{x}}$, M. Nyklicek ${ }^{\mathrm{x}}$, J. Oehlschläger ${ }^{\mathrm{ag}}$, A. Olinto ${ }^{\mathrm{ch}}$, P. Oliva ${ }^{\text {af }}$, V.M. Olmos-Gilbaja ${ }^{\text {br }}$, M. Ortiz ${ }^{\text {bo }}$, N. Pacheco ${ }^{\text {bp }}$, D. Pakk Selmi-Dei ${ }^{\circ}$, M. Palatka ${ }^{\mathrm{x}}$, J. Pallotta ${ }^{\mathrm{c}}$, N. Palmieri ${ }^{\text {ai }}$, G. Parente ${ }^{\text {br }}$, E. Parizot ${ }^{\text {aa }}$, A. Parra ${ }^{\text {br }}$, J. Parrisius ${ }^{\text {ai }}$, R.D. Parsons ${ }^{\text {bt }}$, S. Pastor ${ }^{\text {bn }}$, T. Paul $^{\text {cd }}$, M. Pech ${ }^{\mathrm{x}}$, J. Pękala ${ }^{\text {bi }}$, R. Pelayo ${ }^{\text {br }}$, I.M. Pepe ${ }^{\mathrm{r}}$, L. Perrone ${ }^{\mathrm{av}}$, R. Pesce $^{\mathrm{an}}$, , E. Petermann $^{\mathrm{ci}}$, S. Petrera $^{\text {ao }}$, P. Petrinca as , A. Petrolini an, Y. Petrov ${ }^{\text {bx }}$, J. Petrovic ${ }^{\text {bg }}$, C. Pfendner ${ }^{c k}$, N. Phan $^{c j}$, R. Piegaia ${ }^{d}$, T. Pierog $^{\text {ag }}$,

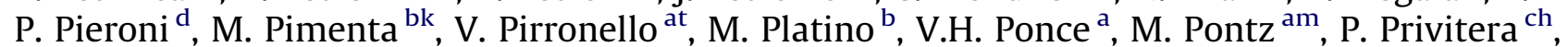

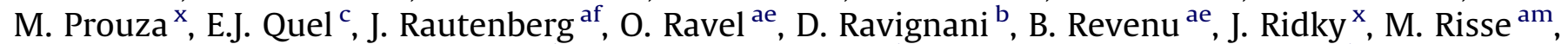
P. Ristori ${ }^{c}, H$. Rivera ${ }^{\text {ap }}$, C. Riviére ${ }^{\text {ad }}$, V. Rizi $^{\text {ao }}$, C. Robledo ${ }^{\text {ba }}$, W. Rodrigues de Carvalho ${ }^{\text {br,n }}$, G. Rodriguez ${ }^{\text {br }}$, J. Rodriguez Martino ${ }^{\text {h,at }}$, J. Rodriguez Rojo ${ }^{\text {, }}$, I. Rodriguez-Cabo ${ }^{\text {br }}$, M.D. RodríguezFrías $^{\text {bp }}$, G. Ros ${ }^{\text {bp }}$, J. Rosado ${ }^{\text {bo }}$, T. Rossler ${ }^{\mathrm{y}}$, M. Roth $^{\mathrm{ag}}$, B. Rouillé-d’Orfeuil ${ }^{\mathrm{ch}}$, E. Roulet ${ }^{\mathrm{a}}$, A.C. Rovero ${ }^{\mathrm{f}}$, C. Rühle ${ }^{\text {ah }}$, F. Salamida ag,ao, H. Salazar ${ }^{\text {ba }}$, G. Salina ${ }^{\text {as }}$, F. Sánchez ${ }^{\text {b }}$, M. Santander ${ }^{\text {h }}$, C.E. Santo ${ }^{\text {bk }}$, E. Santos ${ }^{\text {bk }}$, E.M. Santos ${ }^{\text {t }}$, F. Sarazin ${ }^{\text {bw }}$, S. Sarkar ${ }^{\text {bs }}$, R. Sato ${ }^{\text {h }}$, N. Scharf ${ }^{\text {ak }}$, V. Scherini ${ }^{\text {ap }}$, H. Schieler ${ }^{\text {ag }}$, P. Schiffer ${ }^{\text {ak }}$, A. Schmidt ${ }^{\text {ah }}$, F. Schmidt ${ }^{\text {ch }}$, T. Schmidt ${ }^{\text {ai }}$, O. Scholten ${ }^{\text {bf }}$, H. Schoorlemmer ${ }^{\text {be }}$,

J. Schovancova ${ }^{\mathrm{x}}$, P. Schovánek ${ }^{\mathrm{x}}$, F. Schroeder ${ }^{\text {ag }}$, S. Schulte ${ }^{\mathrm{ak}}$, D. Schuster ${ }^{\text {bw }}$, S.J. Sciutto ${ }^{\text {e }}$, M. Scuderi ${ }^{\text {at }}$, A. Segreto ${ }^{\text {aw }}$, D. Semikoz ${ }^{\text {aa }}$, M. Settimo ${ }^{\text {am,aq }}$, A. Shadkam ${ }^{\text {ca }}$, R.C. Shellard ${ }^{\text {k,l }}$, I. Sidelnik ${ }^{\text {b }}$, G. Sigl $^{\text {al }}$, A. Śmiałkowski ${ }^{\text {bj, R. Šmída ag,x }}{ }^{\text {, G.R. Snow }}{ }^{\text {ci, }}$, P. Sommers ${ }^{\text {cf }}$, J. Sorokin ${ }^{j}$, H. Spinka bu,bz, R. Squartini ${ }^{\text {h }}$,

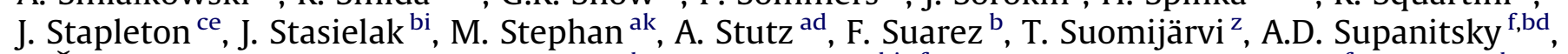

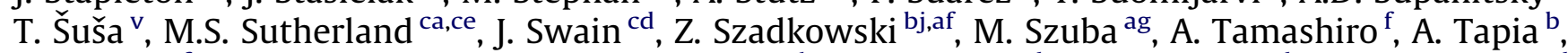
O. Taşcău ${ }^{\text {af }}$, R. Tcaciuc ${ }^{a m}$, D. Tegolo ${ }^{\text {at,az }}$, N.T. Thao ${ }^{1, c m}$, D. Thomas ${ }^{\text {bx }}$, J. Tiffenberg ${ }^{d}$, C. Timmermans ${ }^{\text {bg,be }}$, D.K. Tiwari ${ }^{\text {bc }}$, W. Tkaczyk ${ }^{\text {bj }}$, C.J. Todero Peixoto ${ }^{\mathrm{m}, \mathrm{s}}$, B. Tomé $^{\mathrm{bk}}$, A. Tonachini $^{\text {au }}$, P. Travnicek ${ }^{\mathrm{x}}$, D.B. Tridapalli ${ }^{\text {n }}$, G. Tristram ${ }^{\text {aa }}$, E. Trovato ${ }^{\text {at }}$, M. Tueros ${ }^{\text {br,d }}$, R. Ulrich ${ }^{\text {cf,ag }}$, M. Unger ${ }^{\text {ag }}$, M. Urban ${ }^{\text {ab }}$, J.F. Valdés Galicia ${ }^{\text {bd }}$, I. Valiño ${ }^{\text {br,ag }}{ }^{\text {, L. Valore }}{ }^{\text {ar }}$, A.M. van den Berg ${ }^{\text {bf }}$, B. Vargas Cárdenas ${ }^{\text {bd }}$, J.R. Vázquez ${ }^{\text {bo }}$, R.A. Vázquez ${ }^{\text {br }}$, D. Veberič ${ }^{\text {bm,bb }}{ }^{\text {, V. Verzi }}{ }^{\text {as }}$, M. Videla ${ }^{g}$, L. Villaseñor ${ }^{\text {bc }}$, H. Wahlberg $^{\text {e }}$, P. Wahrlich ${ }^{\mathrm{j}}$, O. Wainberg ${ }^{\mathrm{b}}$, D. Warner ${ }^{\mathrm{bx}}$, A.A. Watson $^{\text {bt }}$, M. Weber $^{\text {ah }}$, K. Weidenhaupt $^{\text {ak }}$, A. Weindl $^{\text {ag }}$, S. Westerhoff ${ }^{\text {ck }}$, B.J. Whelan ${ }^{j}$, G. Wieczorek ${ }^{\text {bj }}$, L. Wiencke ${ }^{\text {bw }}$, B. Wilczyńska ${ }^{\text {bi }}$, H. Wilczyński ${ }^{\text {bi }}$, M. Will ${ }^{\mathrm{ag}}$, C. Williams ${ }^{\mathrm{ch}}$, T. Winchen ${ }^{\mathrm{ak}}$, L. Winders ${ }^{\mathrm{cl}}$, M.G. Winnick ${ }^{\mathrm{j}}$, M. Wommer $^{\mathrm{ag}}$, B. Wundheiler $^{\mathrm{b}}$, T. Yamamoto ${ }^{\text {ch,2, P. Younk }}{ }^{\text {am,bx }}$, G. Yuan ${ }^{\text {ca }}$, B. Zamorano bq, E. Zas ${ }^{\text {br }}$, D. Zavrtanik ${ }^{\text {bm,bl }}$, M. Zavrtanik ${ }^{\text {bl,bm' }}$ I. Zaw ${ }^{\text {cc }}$, A. Zepeda ${ }^{\text {bb }}$, M. Ziolkowski ${ }^{\text {am }}$

\footnotetext{
${ }^{a}$ Centro Atómico Bariloche and Instituto Balseiro (CNEA-UNCUyo-CONICET), San Carlos de Bariloche, Argentina

${ }^{\mathrm{b}}$ Centro Atómico Constituyentes (Comisión Nacional deEnergía Atómica/CONICET/UTN-FRBA), Buenos Aires, Argentina

${ }^{c}$ Centro de Investigaciones en Láseres y Aplicaciones, CITEFA and CONICET, Argentina

d Departamento de Física, FCEyN, Universidad de BuenosAires y CONICET, Argentina

e IFLP, Universidad Nacional de La Plata and CONICET, LaPlata, Argentina

${ }^{\mathrm{f}}$ Instituto de Astronomía y Física del Espacio (CONICET-UBA), Buenos Aires, Argentina

${ }^{g}$ National Technological University, Faculty Mendoza (CONICET/CNEA), Mendoza, Argentina

${ }^{\text {h }}$ Pierre Auger Southern Observatory, Malargüe, Argentina

${ }^{i}$ Pierre Auger Southern Observatory and Comisión Nacionalde Energía Atómica, Malargüe, Argentina

j University of Adelaide, Adelaide, S.A., Australia

${ }^{\mathrm{k}}$ Centro Brasileiro de Pesquisas Fisicas, Rio de Janeiro, RJ, Brazil

${ }^{1}$ Pontifícia Universidade Católica, Rio de Janeiro, RJ, Brazil

${ }^{\mathrm{m}}$ Universidade de São Paulo, Instituto de Física, São Carlos, SP, Brazil

${ }^{n}$ Universidade de São Paulo, Instituto de Física, São Paulo, SP, Brazil

${ }^{\circ}$ Universidade Estadual de Campinas, IFGW, Campinas, SP, Brazil

${ }^{\mathrm{P}}$ Universidade Estadual de Feira de Santana, Brazil

${ }^{\mathrm{q}}$ Universidade Estadual do Sudoeste da Bahia, Vitoria da Conquista, BA, Brazil

${ }^{\mathrm{r}}$ Universidade Federal da Bahia, Salvador, BA, Brazil

${ }^{s}$ Universidade Federal do ABC, Santo André, SP, Brazil

${ }^{\mathrm{t}}$ Universidade Federal do Rio de Janeiro, Instituto de Física, Rio de Janeiro, RJ, Brazil

u Universidade Federal Fluminense, Instituto de Fisica, Niterói, RJ, Brazil

${ }^{v}$ Rudjer Bošković Institute, 10000 Zagreb, Croatia

${ }^{\mathrm{w}}$ Charles University, Faculty of Mathematics and Physics, Institute of Particle and Nuclear Physics, Prague, Czech Republic

${ }^{\mathrm{x}}$ Institute of Physics of the Academy of Sciences of the Czech Republic, Prague, Czech Republic

${ }^{y}$ Palacky University, RCATM, Olomouc, Czech Republic

${ }^{\mathrm{z}}$ Institut de Physique Nucléaire d'Orsay (IPNO), Université Paris 11, CNRS-IN2P3, Orsay, France

aa Laboratoire AstroParticule et Cosmologie (APC), Université Paris 7, CNRS-IN2P3, Paris, France

${ }^{\mathrm{ab}}$ Laboratoire de l'Accélérateur Linéaire (LAL), Université Paris 11, CNRS-IN2P3, Orsay, France

ac Laboratoire de Physique Nucléaire et de Hautes Energies (LPNHE), Universités Paris 6 et Paris 7, CNRS-IN2P3, Paris, France

ad Laboratoire de Physique Subatomique et de Cosmologie (LPSC), Université Joseph Fourier, INPG, CNRS-IN2P3, Grenoble, France

ae SUBATECH, CNRS-IN2P3, Nantes, France

af Bergische Universität Wuppertal, Wuppertal, Germany

ag Karlsruhe Institute of Technology - Campus North - Institut für Kernphysik, Karlsruhe, Germany

${ }^{\text {ah }}$ Karlsruhe Institute of Technology - Campus North - Institut für Prozessdatenverarbeitung und Elektronik, Karlsruhe, Germany

ai Karlsruhe Institute of Technology - Campus South - Institut für Experimentelle Kernphysik (IEKP), Karlsruhe, Germany
} 
aj Max-Planck-Institut für Radioastronomie, Bonn, Germany

${ }^{\mathrm{ak}}$ RWTH Aachen University, III. Physikalisches Institut A, Aachen, Germany

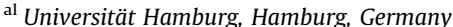

am Universität Siegen, Siegen, Germany

an Dipartimento di Fisica dell'Università and INFN, Genova, Italy

ao Università dell'Aquila and INFN, L'Aquila, Italy

ap Università di Milano and Sezione INFN, Milan, Italy

${ }^{\text {aq }}$ Dipartimento di Fisica dell'Università del Salento and Sezione INFN, Lecce, Italy

ar Università di Napoli "Federico II" and Sezione INFN, Napoli, Italy

as Università di Roma II "Tor Vergata" and Sezione INFN, Roma, Italy

at Università di Catania and Sezione INFN, Catania, Italy

au Università di Torino and Sezione INFN, Torino, Italy

av Dipartimento di Ingegneria dell'Innovazionedell'Università del Salento and Sezione INFN, Lecce, Italy

${ }^{\mathrm{aw}}$ Istituto di Astrofisica Spaziale e Fisica Cosmica di Palermo (INAF), Palermo, Italy

ax Istituto di Fisica dello Spazio Interplanetario (INAF), Università di Torino and Sezione INFN, Torino, Italy

ay INFN, Laboratori Nazionali del Gran Sasso, Assergi (L'Aquila), Italy

${ }^{\mathrm{az}}$ Università di Palermo and Sezione INFN, Catania, Italy

ba Benemérita Universidad Autónoma de Puebla, Puebla, Mexico

bb Centro de Investigación y de Estudios Avanzados del IPN (CINVESTAV), México, D.F., Mexico

bc Universidad Michoacana de San Nicolas de Hidalgo, Morelia, Michoacan, Mexico

${ }^{\text {bd }}$ Universidad Nacional Autonoma de Mexico, Mexico, D.F., Mexico

be IMAPP, Radboud University, Nijmegen, Netherlands

${ }^{\text {bf }}$ Kernfysisch Versneller Institute, University of Groningen, Groningen, Netherlands

bg NIKHEF, Amsterdam, Netherlands

${ }^{\text {bh }}$ ASTRON, Dwingeloo, Netherlands

bi Institute of Nuclear Physics PAN, Krakow, Poland

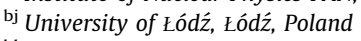

${ }^{\mathrm{bk}}$ LIP and Instituto Superior Técnico, Lisboa, Portugal

bl J. Stefan Institute, Ljubljana, Slovenia

${ }^{\mathrm{bm}}$ Laboratory for Astroparticle Physics, University of Nova Gorica, Slovenia

bn Instituto de Física Corpuscular, CSIC-Universitat de València, Valencia, Spain

bo Universidad Complutense de Madrid, Madrid, Spain

bp Universidad de Alcalá, Alcalá de Henares, Madrid, Spain

bq Universidad de Granada \& C.A.F.P.E., Granada, Spain

${ }^{\mathrm{br}}$ Universidad de Santiago de Compostela, Spain

bs Rudolf Peierls Centre for Theoretical Physics, University of Oxford, Oxford, United Kingdom

bt School of Physics and Astronomy, University of Leeds, United Kingdom

bu Argonne National Laboratory, Argonne, IL, USA

bv Case Western Reserve University, Cleveland, $\mathrm{OH}, \mathrm{USA}$

${ }^{\text {bw }}$ Colorado School of Mines, Golden, CO, USA

bx Colorado State University, Fort Collins, CO, USA

by Colorado State University, Pueblo, CO, USA

${ }^{\text {bz }}$ Fermilab, Batavia, IL, USA

ca Louisiana State University, Baton Rouge, LA, USA

${ }^{\mathrm{cb}}$ Michigan Technological University, Houghton, MI, USA

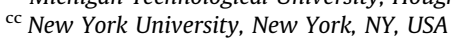

${ }^{\mathrm{cd}}$ Northeastern University, Boston, MA, USA

ce Ohio State University, Columbus, $\mathrm{OH}$, USA

cf Pennsylvania State University, University Park, PA, USA

${ }^{\mathrm{cg}}$ Southern University, Baton Rouge, LA, USA

ch University of Chicago, Enrico Fermi Institute, Chicago, IL, USA

${ }^{\mathrm{ci}}$ University of Nebraska, Lincoln, NE, USA

${ }^{\mathrm{cj}}$ University of New Mexico, Albuquerque, NM, USA

${ }^{\mathrm{ck}}$ University of Wisconsin, Madison, WI, USA

${ }^{\mathrm{cl}}$ University of Wisconsin, Milwaukee, WI, USA

${ }^{\mathrm{cm}}$ Institute for Nuclear Science and Technology (INST), Hanoi, Vietnam

\section{A R T I C L E I N F O}

\section{Article history:}

Received 3 December 2010

Received in revised form

11 January 2011

Accepted 11 January 2011

Available online 22 January 2011

Keywords:

Cosmic rays

Radio detection

Analysis software

Detector simulation

\section{A B S T R A C T}

The advent of the Auger Engineering Radio Array (AERA) necessitates the development of a powerful framework for the analysis of radio measurements of cosmic ray air showers. As AERA performs "radiohybrid" measurements of air shower radio emission in coincidence with the surface particle detectors and fluorescence telescopes of the Pierre Auger Observatory, the radio analysis functionality had to be incorporated in the existing hybrid analysis solutions for fluorescence and surface detector data. This goal has been achieved in a natural way by extending the existing Auger Offline software framework with radio functionality. In this article, we lay out the design, highlights and features of the radio extension implemented in the Auger Offline framework. Its functionality has achieved a high degree of sophistication and offers advanced features such as vectorial reconstruction of the electric field, advanced signal processing algorithms, a transparent and efficient handling of FFTs, a very detailed simulation of detector effects, and the read-in of multiple data formats including data from various

\footnotetext{
* Corresponding author.

E-mail address: auger_pc@fnal.gov (K.H. Kampert).

1 Deceased.

2 At Konan University, Kobe, Japan.
} 
radio simulation codes. The source code of this radio functionality can be made available to interested parties on request.

(c) 2011 Elsevier B.V. All rights reserved.

\section{Introduction}

Forty years after the initial discovery of radio emission from extensive air showers [1], the CODALEMA [2] and LOPES [3] experiments have re-ignited very active research activities in the field of radio detection of cosmic ray air showers. Nowadays, the field is in a phase of transition from first-generation experiments covering an area of less than $0.1 \mathrm{~km}^{2}$ to large-scale arrays of tens of $\mathrm{km}^{2}$. In particular, the Auger Engineering Radio Array (AERA) [4] will complement the southern site of the Pierre Auger Observatory [5] with 161 autonomous radio detector stations covering an area of $\approx 20 \mathrm{~km}^{2}$.

One particular merit of the Pierre Auger Observatory is its hybrid mode of observation, which uses coincident detection of extensive air showers with both optical fluorescence telescopes (FD) and surface particle detectors (SD) to gain in-depth information on the measured air showers. Consequently, the analysis software has to support complete hybrid processing and interpretation of the data. This requirement is fulfilled by the Auger Offline software framework [6]. To take full advantage of the radio data taken in the hybrid environment of the Pierre Auger Observatory, it is clear that radio analysis functionality, which has so far been existing in a separate software package [7], also had to be included in this hybrid analysis framework.

In this article, we describe how we have therefore built advanced radio analysis functionality into the Auger Offline software framework. The general structure of the radio implementation in the Offline framework will be discussed in Section 2. A number of innovative features have been realized in this context for the very first time. These and other highlights will be discussed in Section 3. Finally, in Section 4 we demonstrate how the advanced radio functionality embedded in the Offline framework can be used to carry out a complete detector simulation and event reconstruction on the basis of a simulated radio event.

\section{Embedding radio functionality in the Offline framework}

The Offline framework has a clear structure to allow for easy maintenance and ongoing shared development over the whole life-time of the Pierre Auger Observatory [6]. In particular, there is a clear separation between the internal representation of the Detector and the Event. The Detector provides access to all of the relevant detector information such as the positions of detector stations in the field, the hardware associated with these stations, etc. The Event data structures in contrast hold all of the data applying to a specific event, such as ADC traces, but also reconstructed quantities such as the event geometry. There is no direct connection between these two entities. Instead, analysis Modules use the defined interfaces of both the Detector and Event data structures to carry out their specific analysis tasks. No interface exists either between separate analysis modules, which can only propagate their results through the Event data structure. This ensures that dependencies between analysis modules are kept to a minimum and facilitates the replacement of individual modules with alternative implementations, thereby providing a very high degree of flexibility.

Clearly, the radio analysis functionality had to be implemented following the same philosophy. The hierarchical implementation of the radio parts of both the Detector and Event classes in addition to the existing FD- and SD-specific classes is depicted in Fig. 1. In analogy to the hierarchy of Stations and PMTs in the SD functionality, the implementation of the radio data structures has been divided into Stations and Channels. A Station represents one location in the field at which the electric field of the radio waves is measured. Data stored at Station level therefore represents the physical electric field devoid of any detector influence except for the location (and limited observing bandwidth) of the Station. In contrast, Channels represent the individual antenna channels at which the "raw" measurement is performed by an ADC digitizing voltages. This clear separation between Channels and Stations is a very powerful concept and is original to the radio implementation in Offline. We will discuss its significance, among other highlights, in the following section.

\section{Highlights of the radio analysis functionality}

The radio functionality in the Offline framework provides a number of unique features facilitating an advanced radio data analysis. In this section, we will describe some of these highlights.

\subsection{Clear separation of Channel- and Station levels}

When analyzing radio data, one is faced with two different "levels". The Channel level is defined by the detector channels acquiring the raw data. These data consist of time series of samples digitized with a sampling rate adequate for the frequency window of interest. Each sample denotes a scalar quantity such as an ADC count recorded by the channel ADC. Low-level detector effects such as the correction for the frequency-dependent response of cables, filters and amplifiers are treated on this level for each Channel individually. Likewise, detector-related studies such as the evaluation of trigger efficiencies would be typically performed on Channel level. When reading in measured data files, the raw data (ADC counts) are filled into the appropriate Channel data structures.

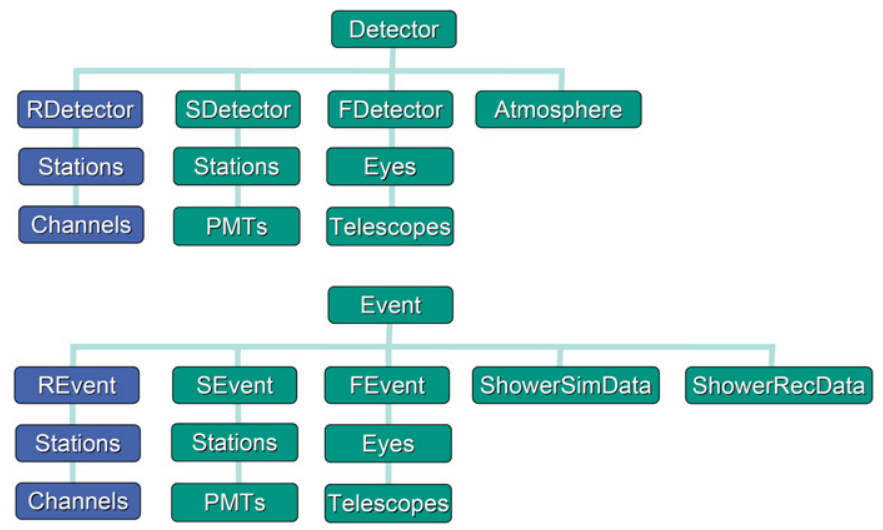

Fig. 1. Within Offline, the detector and event data structures are clearly separated. Both data structures have been complemented with the analogous classes for radio detection (marked in blue). In both the RDetector and the REvent, classes for Stations and Channels are present. Those in the detector data structures provide access to the detector description, the ones in the event data structures store data applying to specific radio events. (For interpretation of the references to color in this figure legend, the reader is referred to the web version of this article.) 
In contrast, the Station level is defined by the physical electric field present at a given location in the field, stored as a time series of three-dimensional vectors. It is on Station level that radio pulses are identified and quantified, before a geometry reconstruction of the given event is performed. Once the event reconstruction has been completed, the data at Station level no longer have any dependence on the detector characteristics, except for the location and limited observing bandwidth of the measurement. A reconstruction of the electric field on the Station level is therefore suited best for a comparison of radio measurements of different experiments, as well as for the comparison of radio measurements with corresponding simulations. Since simulated electric field traces provided by radio emission models also represent physical electric fields independent of a given detector, they are read in on the Station level.

Analysis modules in Offline usually work on either Channel or Station level, and typically it is very clear which analysis step has to be performed on which level. The transition between the two levels is performed by applying the characteristics of the antennas associated to each of the Channels. This transition can be employed in both directions, from Station to Channel or viceversa. The transition from a Station to the associated Channels is typically performed to calculate the response of the individual detector Channels to an electric field provided by simulations. The opposite transition is required when reconstructing the threedimensional electric field vector from the data recorded by the (typically) two measurement channels in the field. This reconstruction will be further discussed in Section 3.7.

\subsection{Read-in from different data sources}

The Event data structures are complemented with reader functionality to populate them with data available in one of several file formats for both experimentally measured data and simulated radio event data. Due to its wealth of supported formats and the possibility of easy extension with new formats, the radio functionality in Offline therefore provides very powerful functionality to compare data and simulations from different sources, which again is an original feature usually not found in the analysis software suites developed in the contexts of other experiments. At the time of writing, the following data formats are supported. For experimental data:

- measurement data from two different prototype setups situated at the Balloon Launching Station of the Pierre Auger Observatory $[8,9]$;

- measurement data from AERA [4].

For simulation data, the following formats are currently readable:

- simulation data from MGMR [10];

- simulation data from REAS2 and REAS3 [11,12];

- simulation data from ReAIRES [13].

\subsection{Modular approach}

The strict interface design of the Detector, the Event and the analysis modules allows for a very modular implementation of radio analysis functionality. As the analysis modules are the part of the code typically the most exposed to the end-user, their interface has been kept relatively simple. End-users developing analysis functionality for Offline therefore only need relatively basic proficiency in $\mathrm{CH}$.

An analysis application within Offline is defined through a "module sequence" in XML syntax, an example of which is listed in Section 4. In such a module sequence, analysis modules are chained in a meaningful sequence to perform a specific analysis task. The individual modules do not communicate directly with each other, but only share data through the Event data structures. Consequently, modules can easily be removed, replaced or rearranged within a module sequence. This does not require recompilation of the source code. Additionally, each module can be configured individually through XML files.

\subsection{Transparent FFT handling}

Radio analyses typically apply algorithms both on time- and frequency-domain data. As a consequence, they heavily rely on fast Fourier transforms (FFTs). The Offline framework has thus been extended with FFT functionality based on the FFTW library [14]. A special feature of this implementation is that FFTs are handled completely transparently in the background. The user does not need to invoke FFTs manually.

This is realized by the use of FFTDataContainers as illustrated in Fig. 2. These containers encapsulate both the time- and frequencydomain representations of radio data on the Channel and Station levels. The user can access both the time-domain and frequencydomain data at any time. The FFTDataContainer keeps track of which representation has been changed last and whether an FFT has to be performed or not before the data requested by the user are returned. All data are passed by reference and changed in place, so that even traces with an extreme length can be handled efficiently.

As a consequence of this design, the user can simply chain analysis modules working in any of the two domains without worrying which domain has last been worked on. (There is a performance benefit when grouping modules working in the same domain together, but it is not very significant.)

\subsection{Advanced analysis modules}

A number of analysis modules performing recurring steps in advanced radio analysis pipelines are available by default. They can easily be included or excluded from module sequences as needed:

- modules applying bandpass filters to the Channel and Station levels;

- a module performing an up-sampling of under-sampled data;

- a module resampling data to a different time-base;

- a module suppressing narrow-band radio frequency interference through a "median filter";

- a module performing an enveloping of time traces via a Hilbert transform;

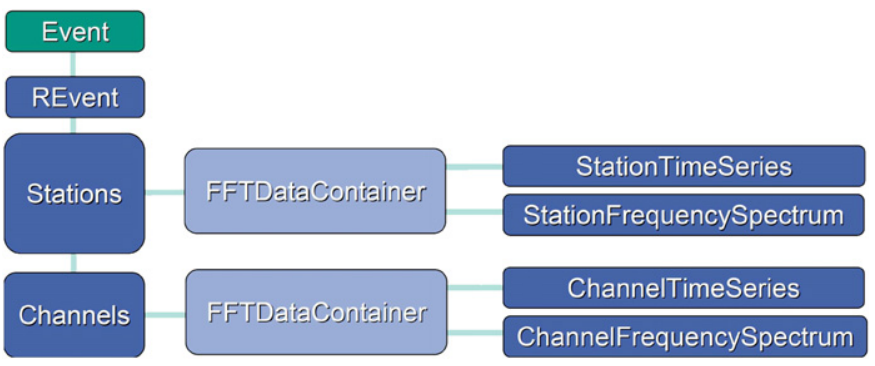

Fig. 2. At both the Station and Channel levels of the REvent, data structures exist to store time series and frequency-domain data. These are encapsulated in FFTDataContainers which transparently and efficiently handle all necessary FFTs without explicit interaction from the end-user. 


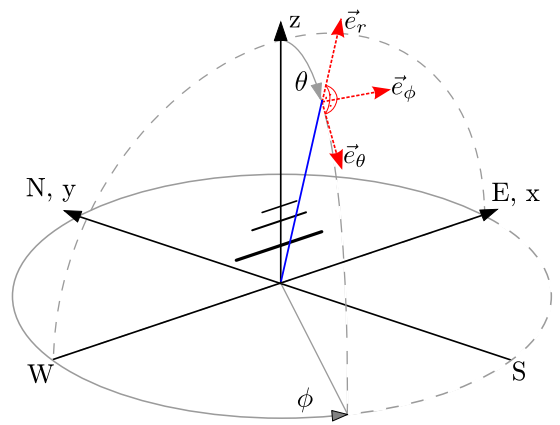

Fig. 3. The antenna characteristics are defined in a spherical coordinate system with the antenna in its center. The effective antenna height $\vec{H}_{\text {eff }}$ for emission coming from a given arrival direction is decomposed into the components along the unit vectors $e_{\theta}$ and $e_{\phi}$. These local (i.e., arrival direction-dependent) unit vectors lie in the plane perpendicular to the Poynting vector, which aligns with the dashed blue line in this figure for the incoming direction defined by $\theta$ and $\phi$. As electromagnetic waves in air have no electric field component along the Poynting vector, this representation is complete. The antenna height itself is a frequencydependent, complex quantity, the amplitude of which denotes the gain of the antenna, while the phase provides information about signal delays and dispersion. (For interpretation of the references to color in this figure legend, the reader is referred to the web version of this article.)

- a module determining timing differences between different antenna stations from the reference phases of a beacon transmitter;

- modules applying a windowing function (e.g., Hann window) to the Channel and Station levels.

\subsection{Detailed simulation of the detector response}

When comparing measured data to simulated radio pulses from various models, it is required to perform a detailed simulation of the effects introduced by the various detector components. This encompasses in particular:

- the complex response (impulse response defined by the frequency-dependent amplitudes and phases ${ }^{3}$ ) of all the analogue components (cables, filters, amplifiers) in each individual channel;

- the frequency- and direction-dependent complex gain (or "effective antenna height") of the antenna connected to each individual channel (cf. Fig. 3);

- effects introduced by the sampling of the data with a given sampling rate;

- saturation effects occurring at the ADCs;

- effects introduced by the layout of the array, including geometric effects occurring on large scales due to the curvature of the Earth.

All of this functionality has been implemented in the Offline framework. At the moment, detector description data are provided as XML files. Later, a transition to MySQL or SQLite databases is foreseen and can be performed transparently. The complex response of individual Channels is provided via a ResponseMap detailing the hardware elements comprising each individual channel. The overall response of each channel is then calculated on-the-fly from the tabulated responses of each

\footnotetext{
${ }^{3}$ A full transport matrix representing the transmission in forward direction, transmission in backward direction, as well as the reflections on the input and output could be implemented for a more detailed description. For the moment, however, we assume that impedance matching in the experimental setup is sufficiently good so that transmission in the forward direction describes the detector response with good precision.
}

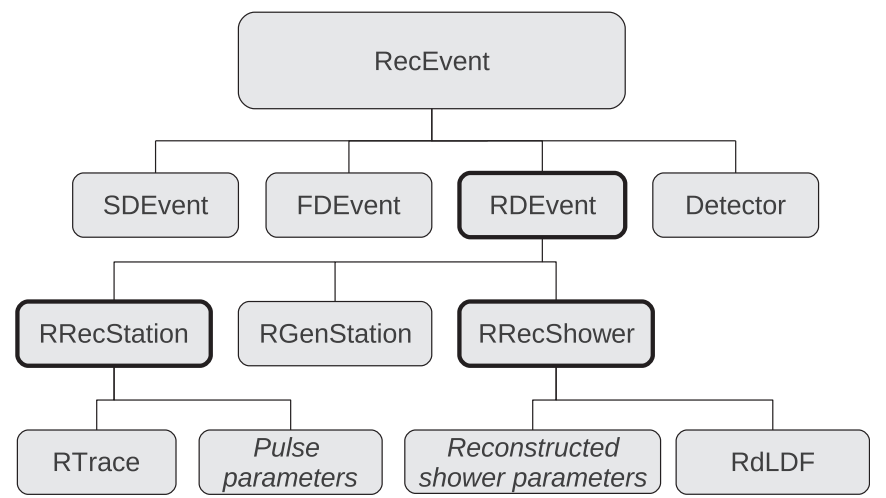

Fig. 4. Hierarchical structure of the radio-related quantities stored in ADST files. The individual classes constitute logical entities present in the Event data structures, storing for example information on Monte Carlo generated quantities in RGenStation and the reconstructed radio lateral distribution function in RdLDF.

individual hardware component listed in the ResponseMap. A caching mechanism ensures that overall responses are only recalculated when needed.

\subsection{Vectorial E-field reconstruction}

The physical electric field is a three-dimensional, vectorial quantity. When comparing results from different experiments or experimental results and radio emission models, the electric field is the quantity of choice, as in principle it has no dependence on the detector (except for the location at which it was measured and the limited observing bandwidth). Most radio detectors, however, are only equipped with two channels per position in the field, typically measuring the east-west and north-south linear polarization components. In other words, they only measure a projection of the three-dimensional electric field to the horizontal plane. In such a setup, two Channels are available at each detector station, one connected with an east-west-aligned antenna and one connected with a north-south-aligned antenna. The (scalar) response of each individual channel to the incoming electric field can be calculated as the dot product between the electric field vector and the vectorial representation of the effective antenna height. The effective antenna height is related to the gain of the antenna and depends on the arrival direction and frequency (cf. Fig. 3). Consequently, this calculation is best done in the frequency domain.

The more difficult problem is the inverse calculation: the reconstruction of the three-dimensional electric field vector from the two-dimensional measurement. This inversion is possible if the arrival direction of the electromagnetic wave is known, because electromagnetic waves in the atmosphere constitute transverse waves, the electric field of which lies in a plane perpendicular to the direction of propagation. ${ }^{4}$

The antenna characteristics needed to reconstruct the vectorial electric field on the Station level depend on the arrival direction. This arrival direction, however, is not available until after the reconstruction on the basis of the Station level three-dimensional electric field. Therefore, an iterative approach starting with a reasonable initial arrival direction is performed in the radio analysis in Offline. The reconstructed arrival direction quickly converges to its final value, and the vertical component of the electric field can be reconstructed from the two-dimensional

\footnotetext{
${ }^{4}$ At the moment, we assume that the propagation direction of radio emission from extensive air showers can be approximated well with the shower axis.
} 
measurement (cf. Section 4). This reconstruction scheme is truly original to the Offline radio functionality.

\subsection{Advanced data output}

End-users performing a higher-level analysis usually do not need access to all the raw data of each individual event. They rather need information about reconstructed quantities derived by the low-level analysis pipeline. A data format to store such quantities reconstructed by an Offline-based analysis is the Advanced Data Summary Tree (ADST). ADST files hold relevant data for FD, SD and now also radio-reconstructed quantities. The structure of the radio data within the ADST files is depicted in Fig. 4.

In addition to accessing the content of ADST files directly from end-user analysis programs, a graphical user interface exists for browsing the contents of ADST files and visualizing the included events. This EventBrowser has also been complemented with radio-specific functionality, so that also the radio part of the event (such as traces, spectra, a map of the array and event geometry, etc.) can be visualized in a user-friendly and intuitive fashion.

\subsection{Easy extension to hybrid analysis}

One of the main motivations for including radio functionality in the Offline framework was to exploit the hybrid nature of the data acquired within the Pierre Auger Observatory. Handling all detector data within the same analysis software will make it easily possible to develop analysis procedures combining data from SD and radio, FD and radio or SD, FD and radio altogether. This will be possible both for measured data and for simulated events. Developing the "radio-hybrid" analysis strategies is yet a challenge for the future. The technical prerequisites for this development have, however, been successfully provided with the inclusion of radio functionality in the Offline framework.

\section{Example for an analysis pipeline}

To illustrate the flexibility and level of sophistication achieved with the radio functionality in Offline, we discuss here a module sequence processing simulated data with a reconstruction pipeline that incorporates all relevant detector effects. Each line in the

\begin{tabular}{|c|c|}
\hline$\langle$ module $>$ EventFileReaderOG & $\langle/$ module $\rangle$ \\
\hline <module> RdStationAssociator & $\langle/$ module $\rangle$ \\
\hline$<!--\quad c f$. Fig. 6 raw simulated electric fields & $-->$ \\
\hline <module> RdAntennaStationToChannelConverter & $\langle/$ module $\rangle$ \\
\hline <module > RdChannelNoiseGenerator & $\langle/$ module $\rangle$ \\
\hline$<!--\quad c f$. Fig. 7 voltages at antenna foot-poin & $-->$ \\
\hline <module> RdChannelResponseIncorporator & $\langle/$ module $\rangle$ \\
\hline$<!--\quad c f . F i g .8$ voltages at $A D C$ & $-->$ \\
\hline <module> RdChannelResampler & $\langle/$ module $\rangle$ \\
\hline <module> RdChannelTimeSeriesClipper & $\langle/$ module $\rangle$ \\
\hline <module> RdChannelVoltageToADCConverter & $\langle/$ module $\rangle$ \\
\hline$<!--$ signal is now equivalent to raw $A D C$ valu & $-->$ \\
\hline <module> RdChannelADCToVoltageConverter & $\langle/$ module $\rangle$ \\
\hline <module > RdChannelPedestalRemover & $\langle/$ module $\rangle$ \\
\hline <module> RdChannelResponseIncorporator & $\langle/$ module $\rangle$ \\
\hline <module > RdChannelRFISuppressor & $\langle/$ module $\rangle$ \\
\hline <module > RdChannelUpsampler & $\langle/$ module $\rangle$ \\
\hline <module> RdChannelBandpassfilter & $\langle/$ module $\rangle$ \\
\hline$<!--\quad c f$. Fig. 9 voltages after signal process & sing \\
\hline \multicolumn{2}{|l|}{ <loop numTimes="unbounded "> } \\
\hline <module> RdAntennaChannelToStationConverter & $\langle/$ module $\rangle$ \\
\hline <module> RdStationSignalReconstructor & $\langle/$ module $\rangle$ \\
\hline <module> RdDirectionConvergenceChecker & $\langle/$ module $\rangle$ \\
\hline$<$ module $>$ RdPlaneFit & $\langle/$ module $\rangle$ \\
\hline$\langle/ 10 \circ p\rangle$ & \\
\hline <module> RdStationWindowSetter & $\langle/$ module $\rangle$ \\
\hline$<!--\quad c f$. Fig. 10 field strengths in clipped t & $\operatorname{trace} \quad-\rightarrow$ \\
\hline <module> RdStationTimeSeriesWindower & $\langle/$ module $\rangle$ \\
\hline$<!--\quad c f$. Fig. 11 Hann-windowed field strength & $-->$ \\
\hline$\langle$ module $>$ RecDataWriter & $\langle/$ module $\rangle$ \\
\hline
\end{tabular}

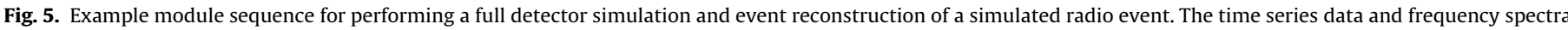

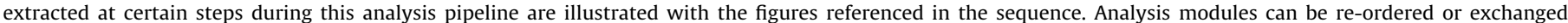
without any recompilation of the source code. 

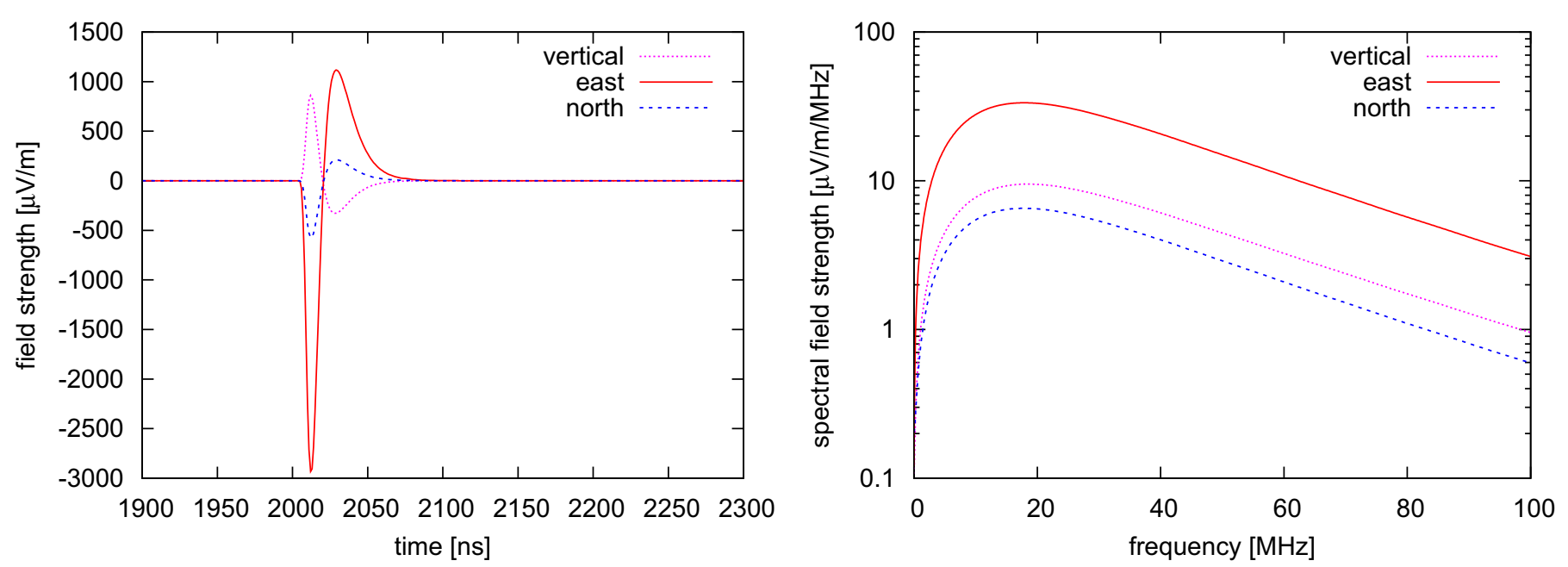

Fig. 6. Time traces (left) and frequency spectra (right) of a simulated event for the raw simulated three-dimensional electric field vector.
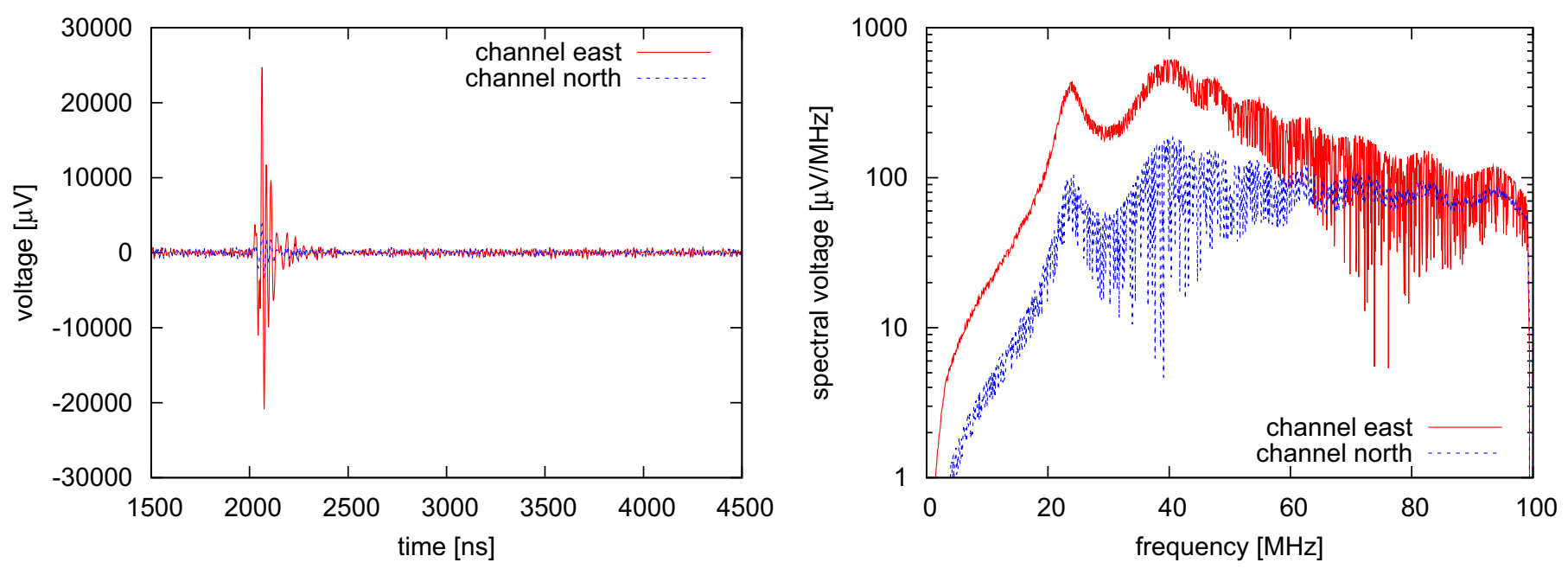

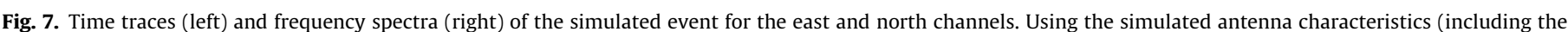

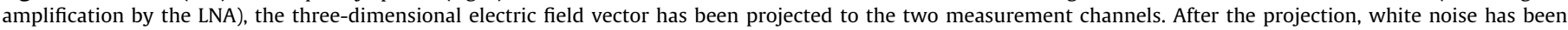

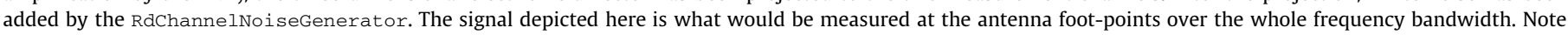
that the frequency spectra correspond to the complete trace and not the zoomed-in time window shown here.

XML file listed in Fig. 5 denotes a module being invoked to perform a specific analysis step. Radio modules starting with RdChannel manipulate the event data on the Channel level, modules starting with RdStation manipulate data on the Station level. The modules starting with RdAntenna perform the transitions between the two levels (cf. Section 3.7). Modules can easily be removed, replaced or rearranged to change the analysis procedure without having to recompile the source code. In the following, we will briefly discuss the individual steps of the module sequence and show how the signal evolves on its way through the analysis pipeline.

\subsection{Read-in and association}

The module sequence starts with the read-in of simulated event data ${ }^{5}$ using the EventFileReaderog module. After readin, the simulated data represent an "abstract" simulation that is not yet associated to any detector stations. This association is

${ }^{5}$ The example event used here has an energy of $2.1 \times 10^{18} \mathrm{eV}$, a zenith angle of $58.4^{\circ}$, an azimuth angle of $291.0^{\circ}$ corresponding to an arrival direction of approximately SSW, and has been simulated with a proton as primary particle. performed by the RdStationAssociatior module, which associates the simulated signal traces with the corresponding stations in the field, and at the same time pads them appropriately to ensure that the signal falls into the correct part of the time series trace. Afterwards, the Station data structure contains the physical electric field vector as predicted by the simulation, without the inclusion of any detector effects. The corresponding traces and spectra are depicted in Fig. 6.

\subsection{Simulation of the detector response}

The next steps in the module sequence change the data such that they become equivalent to data measured experimentally. The RdAntennastationToChannelconverter calculates the signal voltages that each Channel of a given Station would have seen at the foot-points of the corresponding antennas by folding in the antenna response applicable to each individual channel. In the typical case of two antenna Channels per Station, this means that the three-dimensional electric field vector is projected to a twodimensional surface. The RdChannelNoiseGenerator module then adds broad-band radio noise to the event. The resulting, simulated data for the east and north channels are shown in Fig. 7. 
The following call of the RdChannelResponseIncorporator incorporates the (forward) detector response of the cables, filters and amplifiers comprising the corresponding Channel. After this module, the signal represents the voltages that would be measured at the channel ADCs, depicted in Fig. 8.

The following steps convert this voltage at the ADCs to the signal that the channel ADC would indeed have measured. The RdChannelResampler module re-samples the Channel time series data to the time-base with which the data are sampled in the experiment. (The prerequisite to this module is that highfrequency components which could lead to aliasing effects have been suppressed. This is ensured here because the RdChannelResponseIncorporator includes filters that do just that in the experimental setup.) The RdChannelTimeSeriesClipper then clips the Channel traces to the number of samples which are taken in the experimental setup. Finally, the RdChannelvoltageTOADCConverter converts the voltages of each sample to ADC counts that would have been recorded by the channel $A D C s$, thereby taking into account quantization and saturation effects.

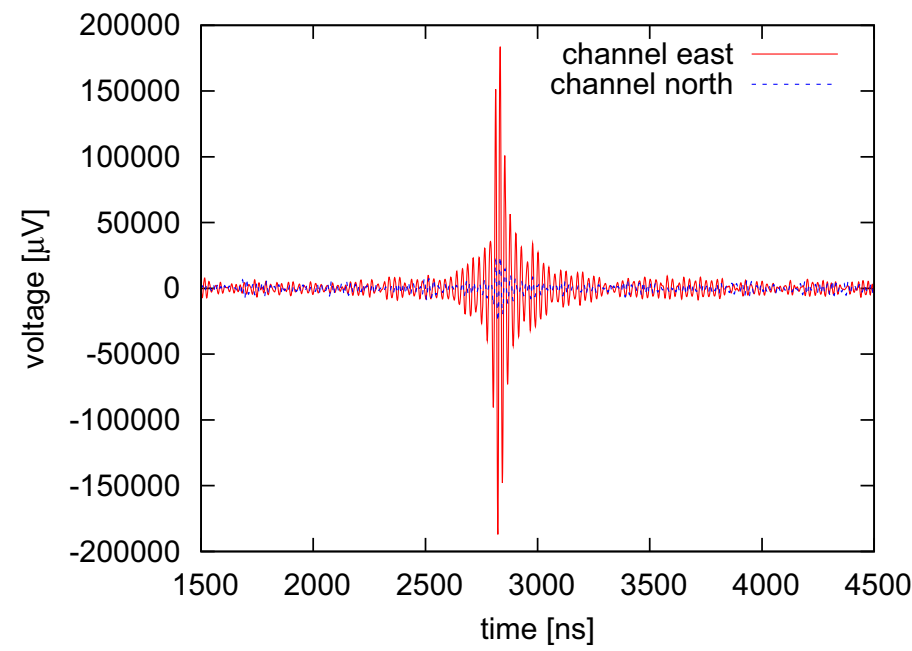

At this point, all of the relevant detector effects have been incorporated in the simulated traces. In other words, the data now have the same properties as measured data directly after read-in. Consequently, the remainder of the reconstruction pipeline is identical to the one that would be applied directly to measured data. Choosing the reconstruction procedure applied to the simulated data identical to the one applied to measured data makes sure that even subtle changes introduced by individual analysis modules can be investigated on the basis of simulations.

\subsection{Signal cleaning}

After converting the ADC counts back to voltages with the RdChannelADCTOVoltageConverter and removing a possible DC offset of the ADC with the RdChannelPedestalRemover, the characteristics of the analogue components of each Channel are folded out from the data with a second call of the RdChannelResponseIncorporator.

The following steps are intended to improve the reconstruction quality by the use of advanced digital processing techniques.

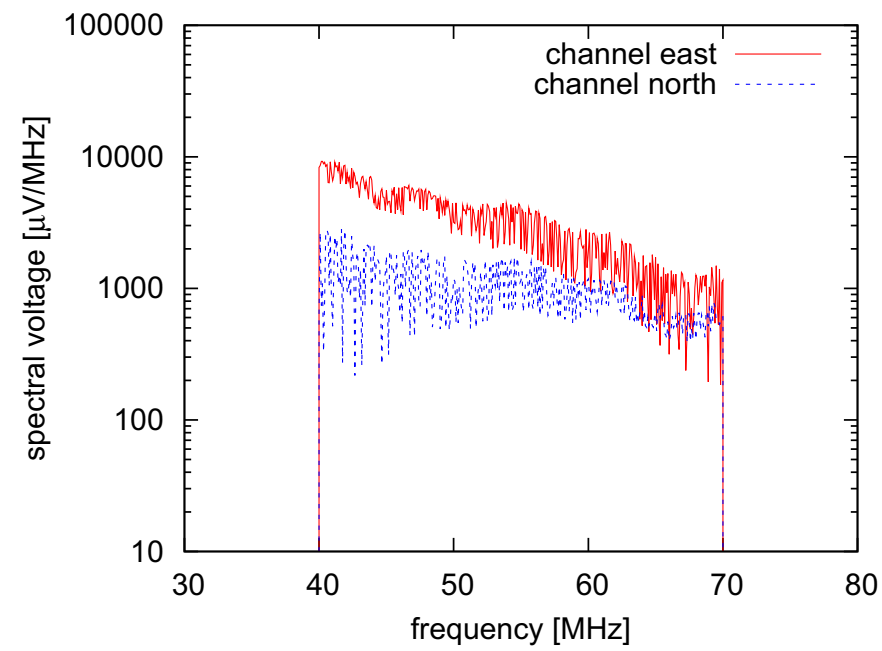

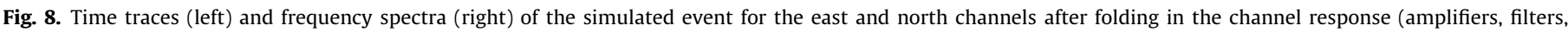

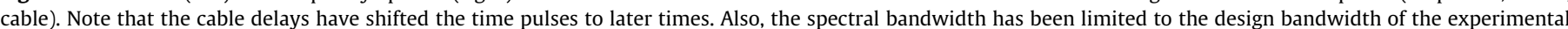

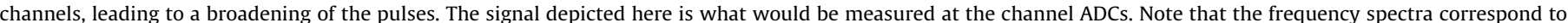
the complete trace and not the zoomed-in time window shown here.
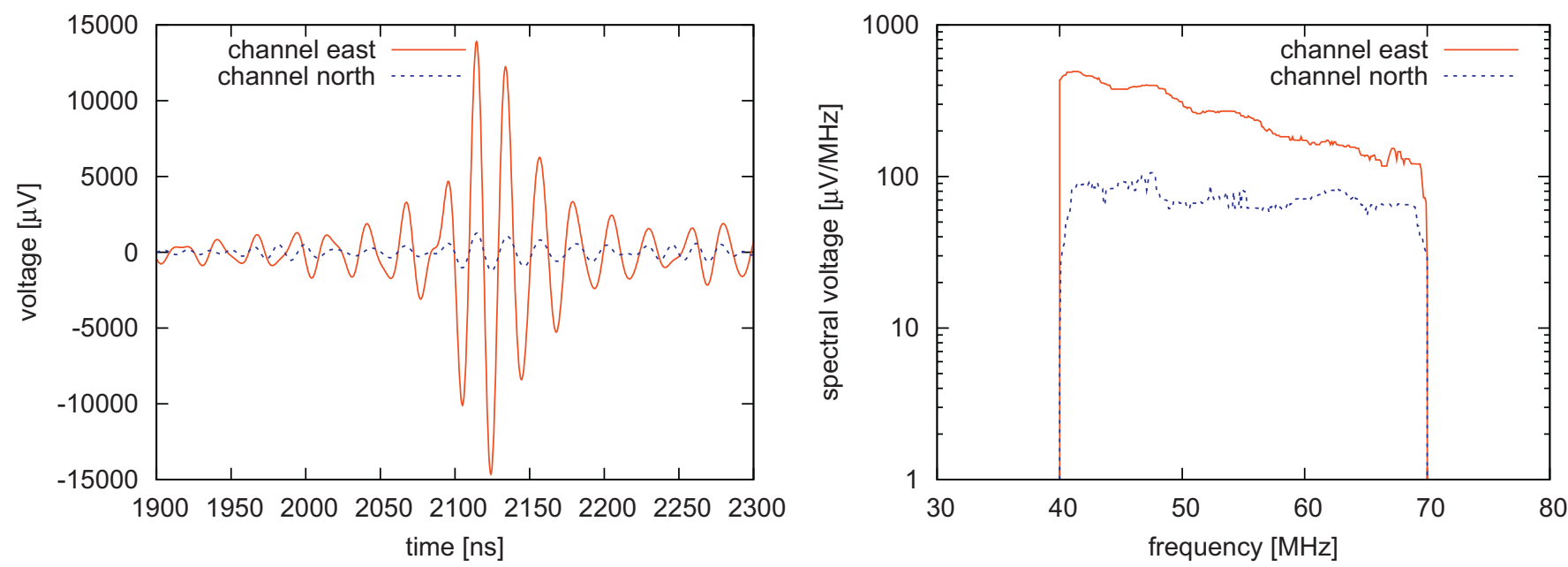

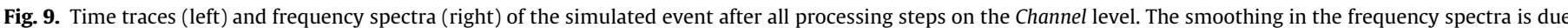

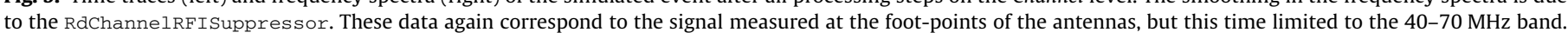

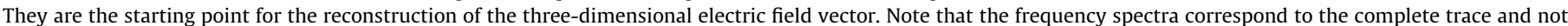
the zoomed-in time window shown here. 
In a first step, the RdChannelRFISuppressor module suppresses narrow-band signals (e.g., TV carriers present in measured data) using a median filter. It is followed by the RdChannelUpsampler module which correctly reconstructs (interpolates) the signal on a finer time-base. (This is possible if the complete signal information is present in the digitized signal, i.e., if the Nyquist criterion for data sampling is fulfilled.) In a further step the signal bandwidth is limited by a digital bandpass filter using the RdChannelBandpassfilter module. After these steps, the signal is ready for the reconstruction of the three-dimensional electric field vector and looks like the data presented in Fig. 9.

\subsection{Vectorial reconstruction}

The following loop performs an iterative reconstruction of the three-dimensional electric field vector and the signal arrival direction. The RdAntennaChannelTostationConverter performs the reconstruction of the three-dimensional electric field described in Section 3.7. Afterwards, the RdStationSignalReconstructor identifies the times at which radio pulses have

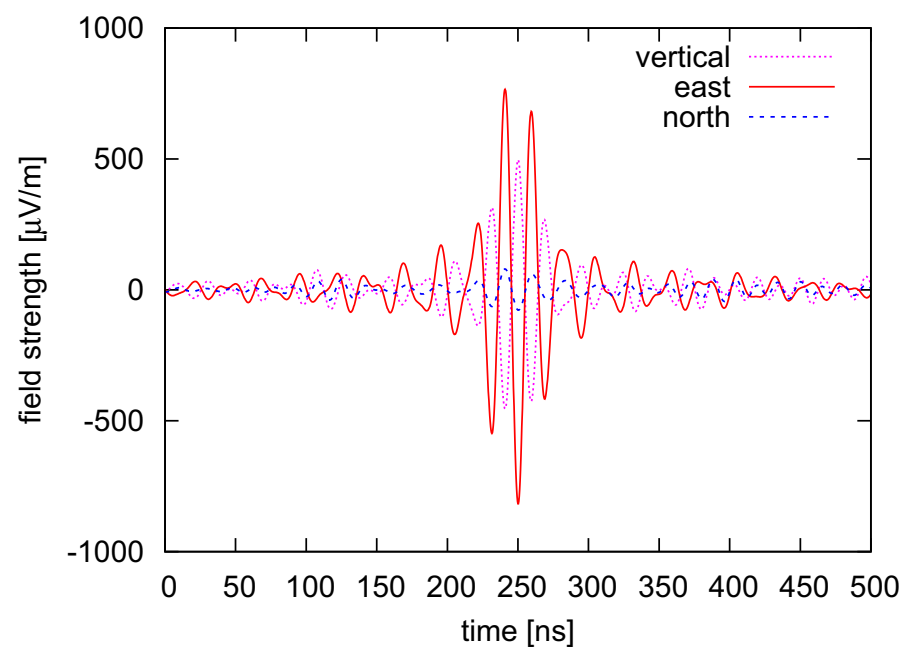

been detected and quantifies the parameters of these pulses. Next, the RdPlaneFit reconstructs the arrival direction of the radio signal with a plane-wave assumption based on the previously established pulse arrival times. Finally, the RaDirectionConvergencechecker module tests whether the iterative procedure has converged or not and breaks the loop accordingly.

\subsection{Post-processing}

After breaking the iterative reconstruction loop, the vectorial, detector-independent electric field has been completely reconstructed. For practical purposes, the time series is then restricted to a window of $500 \mathrm{~ns}$ around the detected pulses with the RdStationwindowsetter. This leads to leakage effects in the frequency spectra, visible in Fig. 10. To suppress these leakage artifacts, a Hann window is applied with the RdStationTimeSerieswindower. The final reconstructed signal is then seen in Fig. 11. As a last step, the RecDataWriter call writes out the reconstructed event data to an ADST file for further processing in higher-level analyses.

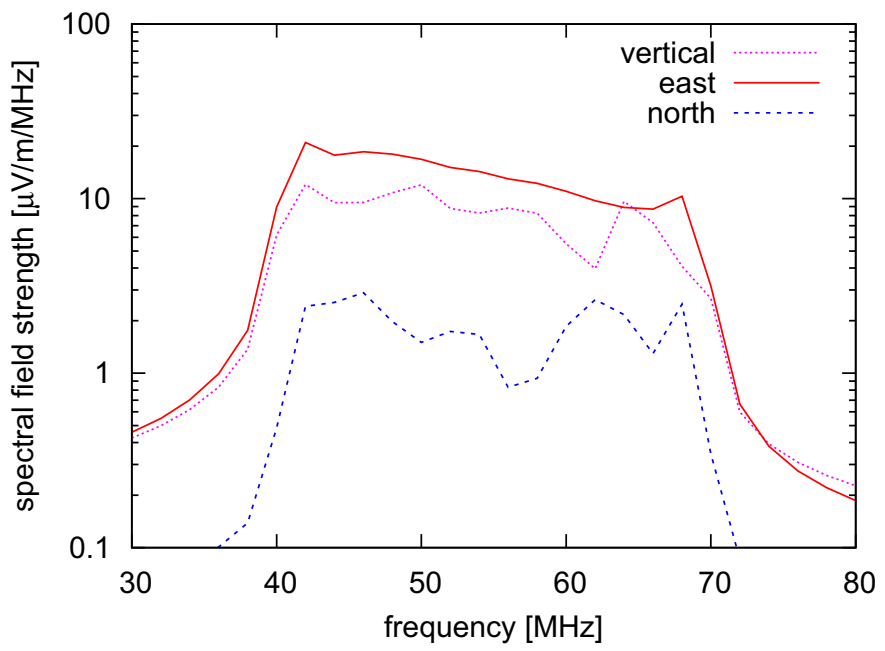

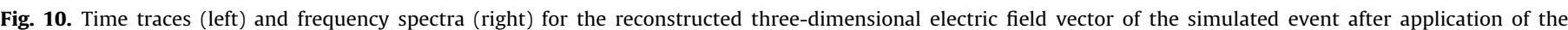

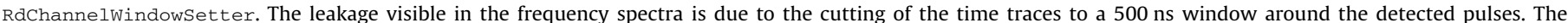
frequency spectra correspond to the time traces shown here.
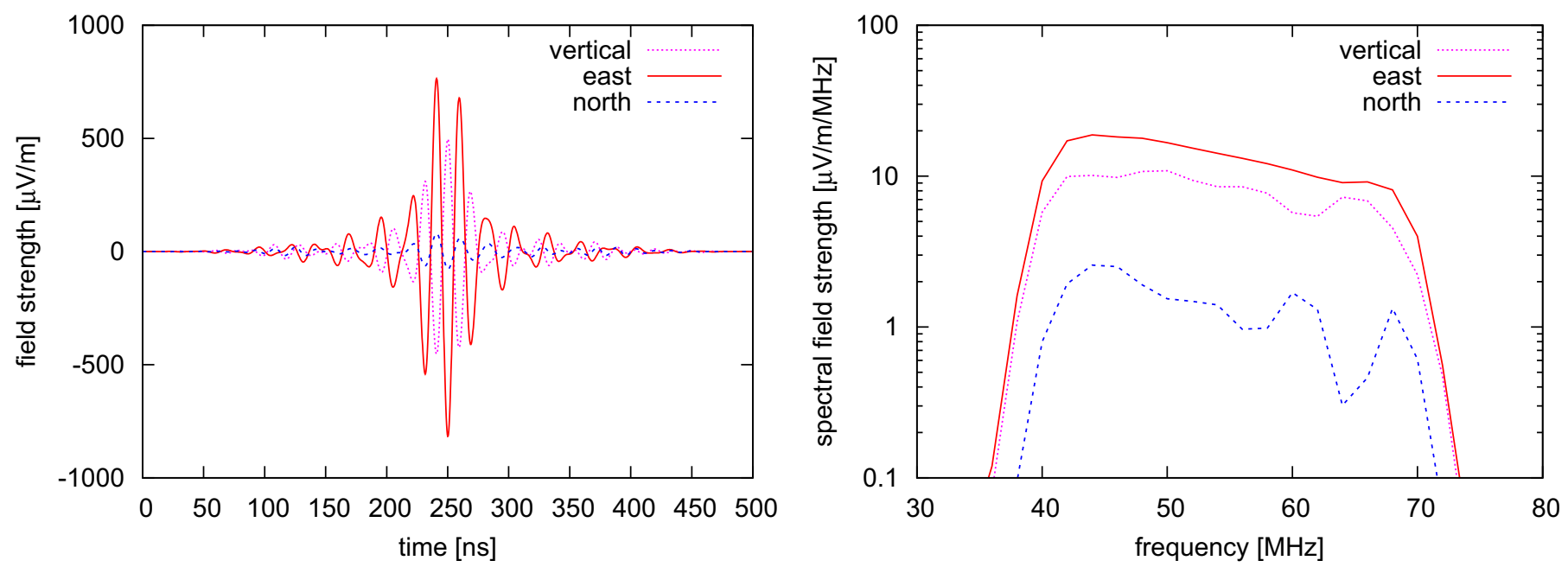

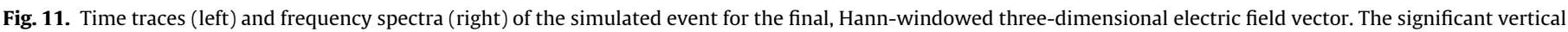

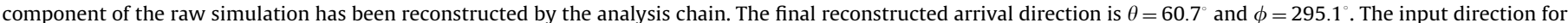
the simulated event was $\theta=58.4^{\circ}$ and $\phi=291.0^{\circ}$. The frequency spectra correspond to the time traces shown here. 


\section{Conclusions and outlook}

We have implemented a complete set of radio analysis functionality in the Offline software framework of the Pierre Auger Observatory. The radio functionality has been included in a canonical and seamless way in addition to the existing SD and FD functionality. This approach will make the realization of "radio-hybrid" analysis strategies in the future straightforward.

Already now, however, the radio functionality in Offline has reached a high degree of sophistication with highlights such as a very fine-grained simulation of detector effects, advanced signal processing algorithms, transparent and efficient handling of FFTs, read-in of multiple file formats for measured and simulated radio data, and in particular the reconstruction of the three-dimensional electric field vector from two-dimensional measurements. Planned improvements encompass the implementation of a curved fit, inclusion of interferometric radio analysis functionality, and the handling of a time-variable detector including a finegrained treatment of the instrumental calibration.

Parties interested in using the functionality are encouraged to contact the corresponding author. The source code can be made available on request.

\section{Acknowledgments}

We would like to thank our colleagues from the LOPES collaboration for providing the source code of their analysis software openly to the public [15]. Many algorithms for radio analysis and reconstruction have been inspired by or based on those used within LOPES. This research has been supported by grant no. VH-NG-413 of the Helmholtz Association.

The successful installation and commissioning of the Pierre Auger Observatory would not have been possible without the strong commitment and effort from the technical and administrative staff in Malargüe.

We are grateful to the following agencies and organizations for financial support: Comisión Nacional de Energía Atómica, Fundación Antorchas, Gobierno De La Provincia de Mendoza, Municipalidad de Malargüe, NDM Holdings and Valle Las Leñas, in gratitude for their continuing cooperation over land access, Argentina; the Australian Research Council; Conselho Nacional de Desenvolvimento Científico e Tecnológico (CNPq), Financiadora de Estudos e Projetos (FINEP), Fundação de Amparo à Pesquisa do Estado de Rio de Janeiro (FAPERJ), Fundação de Amparo à Pesquisa do Estado de São Paulo (FAPESP), Ministério de Ciência e Tecnologia (MCT), Brazil; AVCR, AV0Z10100502 and AV0Z10100522, GAAV KJB300100801 and KJB100100904, MSMT-CR LA08016, LC527, 1M06002, and MSM0021620859, Czech Republic; Centre de Calcul IN2P3/CNRS, Centre National de la Recherche Scientifique (CNRS), Conseil Régional Ile-de-France, Département Physique Nucléaire et
Corpusculaire (PNC-IN2P3/CNRS), Département Sciences de l'Univers (SDU-INSU/CNRS), France; Bundesministerium für Bildung und Forschung (BMBF), Deutsche Forschungsgemeinschaft (DFG), Finanzministerium Baden-Württemberg, Helmholtz-Gemeinschaft Deutscher Forschungszentren (HGF), Ministerium für Wissenschaft und Forschung, Nordrhein-Westfalen, Ministerium für Wissenschaft, Forschung und Kunst, Baden-Württemberg, Germany; Istituto Nazionale di Fisica Nucleare (INFN), Istituto Nazionale di Astrofisica (INAF), Ministero dell'Istruzione, dell'Università e della Ricerca (MIUR), Gran Sasso Center for Astroparticle Physics (CFA), Italy; Consejo Nacional de Ciencia y Tecnología (CONACYT), Mexico; Ministerie van Onderwijs, Cultuur en Wetenschap, Nederlandse Organisatie voor Wetenschappelijk Onderzoek (NWO), Stichting voor Fundamenteel Onderzoek der Materie (FOM), Netherlands; Ministry of Science and Higher Education, Grant nos. 1 P03 D 014 30 and N N202 207238, Poland; Fundação para a Ciência e a Tecnologia, Portugal; Ministry for Higher Education, Science, and Technology, Slovenian Research Agency, Slovenia; Comunidad de Madrid, Consejería de Educación de la Comunidad de Castilla La Mancha, FEDER funds, Ministerio de Ciencia e Innovación and Consolider-Ingenio 2010 (CPAN), Generalitat Valenciana, Junta de Andalucía, Xunta de Galicia, Spain; Science and Technology Facilities Council, United Kingdom; Department of Energy, Contract nos. DEAC02-07CH11359, DE-FR02-04ER41300; National Science Foundation, Grant no. 0969400, The Grainger Foundation USA; ALFA-EC/ HELEN, European Union 6th Framework Program, Grant no. MEIFCT-2005-025057, European Union 7th Framework Program, Grant no. PIEF-GA-2008-220240, and UNESCO.

\section{References}

[1] J.V. Jelley, J.H. Fruin, N.A. Porter, et al., Nature 205 (1965) 327.

[2] D. Ardouin, A. Bellétoile, D. Charrier, et al., Nucl. Instr. and Meth. A 555 (2005) 148.

[3] H. Falcke, W.D. Apel, A.F. Badea, et al., Nature 435 (2005) 313.

[4] T. Huege, for the Pierre Auger Collaboration, Nucl. Instr. and Meth. A 617 (2009) 484

[5] J. Abraham, M. Aglietta, I.C. Aguirre, et al., Nucl. Instr. and Meth. A 523 (2004) 50.

[6] S. Argiró, S.L.C. Barroso, J. Gonzalez, et al., Nucl. Instr. and Meth. A 580 (2007) 1485.

[7] S. Fliescher, the Pierre Auger Collaboration, Nucl. Instr. and Meth. A 604 (2009) S225.

[8] J. Coppens, the Pierre Auger Collaboration, Nucl. Instr. and Meth. A 604 (2009) S41.

[9] B. Revenu, the Pierre Auger and CODALEMA Collaborations, Nucl. Instr. and Meth. A 604 (2009) S37.

[10] O. Scholten, K. Werner, F. Rusydi, Astropart. Phys. 29 (2008) 94

[11] T. Huege, R. Ulrich, R. Engel, Astropart. Phys. 27 (2007) 392.

[12] M. Ludwig, T. Huege, Astropart. Phys. 34 (2011) 438.

[13] M.A. DuVernois, B. Cai, D. Kleckner, Geosynchrotron radio pulse emission from extensive air showers: simulations with AIRES, in: Proceedings of the 29th ICRC, Pune, India, vol. 8, pp. 311-314.

[14] 〈http://www.fftw.org $\rangle$.

[15] 〈http://usg.lofar.org/svn/code/trunk/ >. 